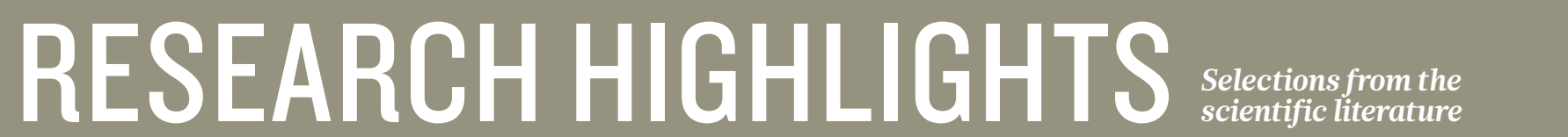

VIROLOGY

\section{Zika shrinks 'mini brains' in culture}

The Zika virus may trigger an immune response that causes developing brain cells to stop dividing and self-destruct.

The link between Zika infection and the birth of babies with abnormally small heads, or microcephaly, has grown stronger, but it is still not clear how the virus attacks developing brains. Tariq Rana at the University of California, San Diego, and his team grew cerebral organoids $-3 \mathrm{D}$ structures that model the developing brain - from human embryonic stem cells and then infected them with Zika. Over a 5-day period, uninfected organoids grew by $22.6 \%$, whereas those exposed to Zika shrank by $16 \%$.

Zika infection boosted the activity of a pathogen-sensing gene, TLR3, which has been linked to brain inflammation and degeneration. Blocking the TLR 3 protein in infected organoids lessened the damage caused by the virus.

Cell Stem Cell http://doi.org/bgrx (2016)

\section{BIOMATERIALS}

\section{Second 'skin'}

\section{turns back time}

A polymer film that sticks to human skin reduces the appearance of wrinkles and bags under the eyes.

Robert Langer at the Massachusetts Institute of Technology in Cambridge and his colleagues designed a polysiloxane-based film that is applied to and cured on the skin. The transparent film has similar mechanical properties to skin, allowing it to conform to the surface. In small studies with human volunteers, the researchers showed that the

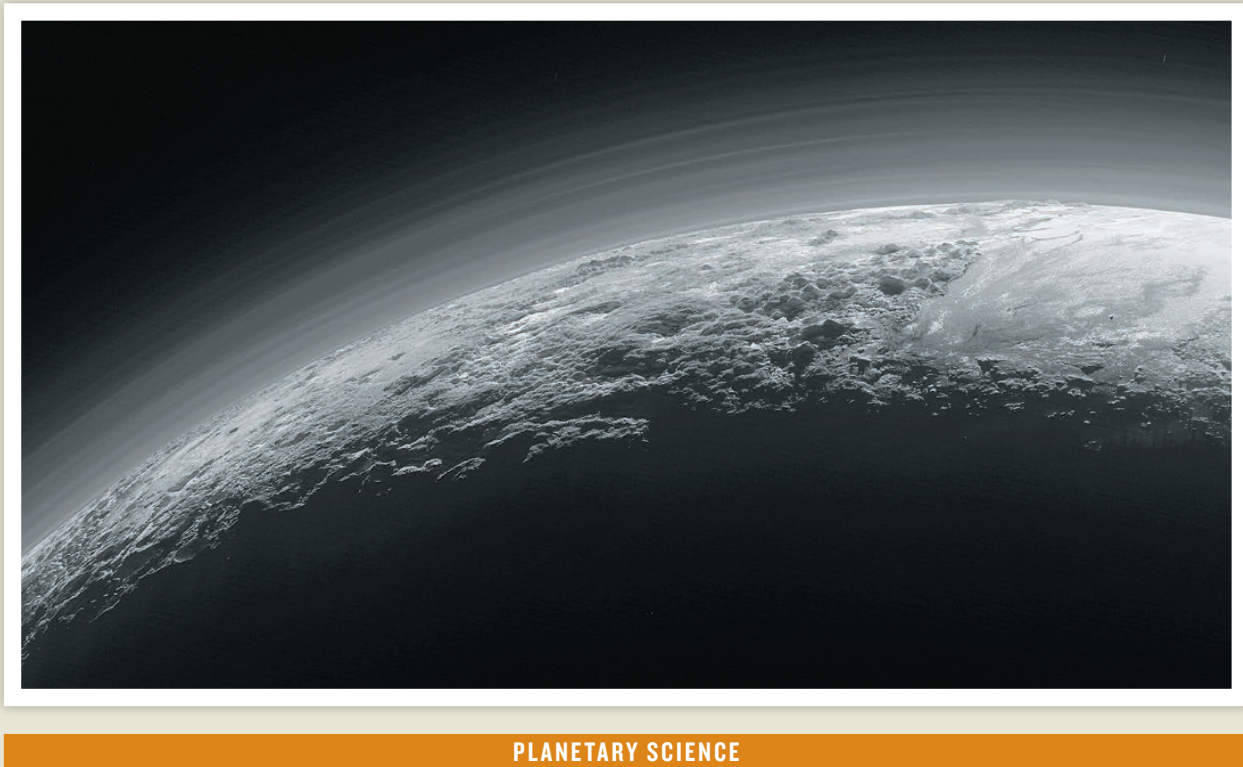

\section{Solar wind hits Pluto hard}

The solar wind is diverted by Pluto, suggesting that, like some larger planets, the dwarf planet has a shield against the stream of energized particles emanating from the Sun.

Before NASA's New Horizons spacecraft visited the dwarf planet (pictured) in 2015, most scientists thought that Pluto interacted with the solar wind in the same way as a comet does. Comets lack protection from the wind, which diffuses around the cometary surface. But in analysing data from the spacecraft, David McComas at Princeton University in
New Jersey and his colleagues identified a 'Plutopause' - a region where Pluto's tenuous atmosphere shields the dwarf planet from the solar wind.

The Plutopause is relatively small and well defined, much like the solar-wind boundaries around Mars and Venus. Even though Pluto is small, it still exerts enough gravitational pull to keep its atmosphere sufficiently close to provide a buffer from the solar wind.

J. Geophys. Res. Space Phys. http://doi.org/bgdv (2016) film reshapes the skin, making bags under the eyes look less puffy and reducing wrinkling.

The film was made from reagents that are considered to be safe for the skin. It could be used cosmetically or in wound dressings, the authors say. Nature Mater. http://dx.doi. org/10.1038/nmat4635 (2016)

BIOPHYSICS

\section{Jammed microbes feel the pressure}

Microbes living in a confined space can push up against each other with enough force to physically damage their environment.

A team led by Oskar Hallatschek of the University of California, Berkeley, created a microscopic chamber that would hold roughly 100 cells of budding yeast (Saccharomyces cerevisiae). As the cells

proliferated, they did not leave the chamber in a steady stream through a narrow exit channel but instead jammed together, building up contact pressures of almost 1 megapascal. This force was enough to cause cracks in agar gels containing growing yeast cells, and to slow down the organism's growth.
Self-driven jamming may help microbes to invade soft materials, which could contribute to biofouling - the accumulation of unwanted microbial material on surfaces, the authors say.

Nature Phys. http://dx.doi. org/10.1038/nphys3741 (2016)

\section{CELL BIOLOGY}

\section{Immune cell aids vascular repair}

White blood cells that gobble up cellular debris also help to heal damaged blood vessels in the brain. 
Deqin Yang at Chongqing Medical University and Lingfei Luo at Southwest University, Beibei in Chongqing, China, and their co-workers used lasers to rupture blood vessels in the brains of zebrafish. They monitored the events that followed with timelapse microscopy, and saw the immune cells, called macrophages, migrate to the damaged area. A macrophage extended projections that adhered to the broken ends of the blood vessels and pulled them together by mechanical traction. In zebrafish engineered to lack macrophages, cerebral blood vessels healed more slowly than in normal animals.

The findings expand on the known roles of macrophages in the brain, the authors say. Immunity http://doi.org/bgrv (2016)

\section{MATERIALS}

\section{Paper strips on the move}

Strips of paper embedded with a conducting polymer can perform a range of movements with electrical stimulation.

George Whitesides at Harvard University in Cambridge, Massachusetts, and his colleagues made

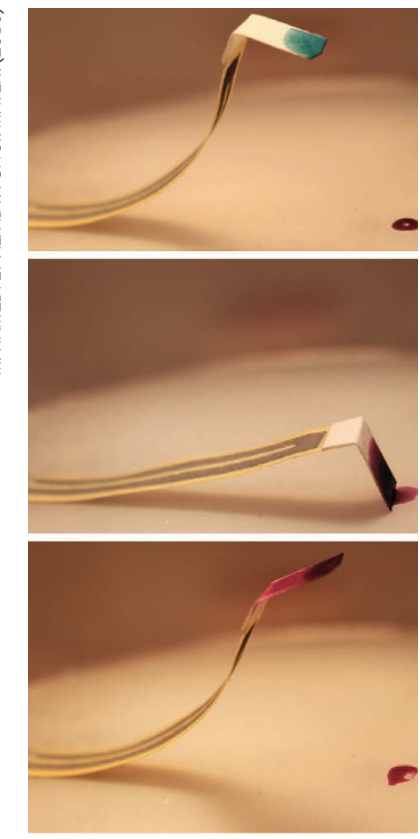

paper actuators that expand and contract according to their water content. They added a conducting polymer that coated the fibres of the paper, and then applied Scotch tape to one side. When electrically activated, the paper heats up, dries out and contracts. When the electrical current is turned off, the paper absorbs water from the air and expands. The tape is not affected by heat or moisture, so directs the paper to bend in certain ways.

The authors made actuators of different shapes, including one that could curl up (pictured), and say that the devices could be used in lightweight micromachines. Adv. Funct. Mater. 26, 2446-2453 (2016)

\section{IMMUNOLOGY \\ How an antibody combats HIV}

A broadly neutralizing antibody against HIV can both boost people's immunity to the virus and directly target infected cells.

The antibody, 3BNC117, has previously been shown to lower HIV levels in the blood of patients. To study its effect on the immune system, Michel Nussenzweig of the Rockefeller University in New York City and his colleagues gave people with HIV one dose of the antibody. They found that patients with higher levels of the virus in their blood developed much broader neutralizing-antibody responses to HIV over six months than did those who had little to no virus (either uninfected individuals or people taking antiretroviral therapies). This indicated that the antibody is boosting the patients' ability to produce other HIV-neutralizing antibodies.

In a second study, a team led by Nussenzweig and Arup Chakraborty of the Massachusetts Institute of Technology in Cambridge showed that the same antibody speeds up the removal of

\section{SOCIAL SELECTION Romituries}

\section{Call calf Higgs Bison, says Twitter}

Just weeks after the public voted overwhelmingly to name a new UK research vessel Boaty McBoatface, the US particle-physics facility Fermilab in Batavia, Illinois, asked people on Twitter to name a bison (pictured) that was born on its grassy grounds on 26 April. "What would you call our new baby bison? Tweet us with \#BisonNaming. Please, no Bison McBisonface." The science world stepped up with a flood of responses - about 260 in the first day. Sandia National Laboratories in Albuquerque, New Mexico, offered "Neil deGrass Bison". Other ideas included Higgs Bison, Enrico Furry

$\begin{array}{ll}\text { FNATURE.COM } & \text { who discovered many } \\ \text { For more on } & \text { radioactive isotopes), } \\ \text { popular papers: } & \text { Bison Tennial and } \\ \text { go.nature.com/kdrhxj } & \text { Niels Bohrson. }\end{array}$

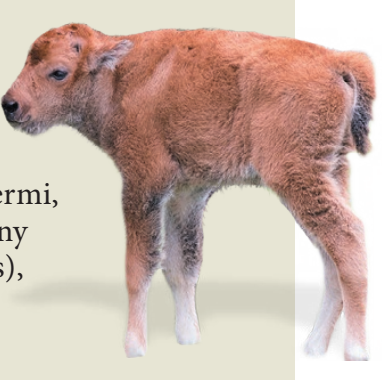

HIV-infected T cells from the blood of mice.

Science http://doi.org/bgdx; http://doi.org/bgdz (2016)

\section{GENETICS \\ CRISPR maps yeast genes}

The CRISPR-Cas9 geneediting system could be harnessed to speed up the search for DNA sequences linked to specific traits.

Researchers can identify genomic regions that are linked to traits, but pinpointing the responsible snippet of DNA within that region is difficult. To speed up the hunt, Meru Sadhu and his colleagues at the University of California, Los Angeles, targeted the Cas 9 enzyme to cut DNA at 95 sites on one copy of chromosome 7 in yeast (Saccharomyces cerevisiae). The team then built a library of yeast strains, each with a genetic rearrangement at one of the 95 sites - making it easier to determine the function of a given section of DNA.

The researchers used their library to pinpoint a gene variant that makes yeast sensitive to manganese. Science http://doi.org/bgd2 (2016)

\section{PLANETARY SCIENCE}

\section{Planet 9 may glow from within}

The hypothetical ninth planet of the Solar System could shine brightly.

Planet 9, if it exists, is thought to be an ice planet that is slightly smaller than Neptune, orbiting in the far outer Solar System. Esther Linder and Christoph Mordasini of the University of Bern in Switzerland modelled the evolution of the planet's probable internal structure. On the basis of its estimated mass and location, they conclude that the planet is still giving off residual heat that was generated when it was formed. This would cause the planet to emit light in the mid- and far-infrared range.

The authors say that future telescopes, such as the Large Synoptic Survey Telescope, or dedicated surveys should be able to detect Planet $9-$ or rule out its existence.

Astron. Astrophys. 589, A134 (2016)

\section{$\rightarrow$ NATURE COM}

For the latest research published by Naturevisit:

www.nature.com/latestresearch 